\title{
Aptamer-switching optical bioassay for citrulline detection at the point-of- care
}

Andrea Locke

Sayali Belsare

Nicolaas Deutz

Gerard Coté 


\title{
Aptamer-switching optical bioassay for citrulline detection at the point-of-care
}

\author{
Andrea Locke, ${ }^{a, b}$ Sayali Belsare, ${ }^{a}$ Nicolaas Deutz, ${ }^{c}$ and Gerard Coté ${ }^{a, b, *}$ \\ ${ }^{a}$ Texas A\&M University, Department of Biomedical Engineering, College Station, Texas, United States \\ ${ }^{\mathrm{b} T e x a s}$ A\&M Engineering Experiment Station Center for Remote Health Technologies and Systems, Department of Biomedical Engineering, \\ College Station, Texas, United States \\ ${ }^{\mathrm{C}}$ Texas A\&M University, Center for Translational Research in Aging and Longevity, Department of Health and Kinesiology, \\ College Station, Texas, United States
}

\begin{abstract}
Researchers have found that decreased levels of circulating citrulline could be an indicator of intestinal failure. Typically, this amino acid, which is produced by the intestinal mucosa cells, circulates in the blood at a physiological level of $\sim 40 \mu \mathrm{M}$. The current methodology for measuring this level involves the use of bulky equipment, such as mass spectroscopy and analysis at a central laboratory, which can delay diagnosis. Therefore, the current detection method is unsuited for routine monitoring at a doctor's office. Our research group proposes the development of a point-of-care (POC) device to overcome this issue. The proposed device utilizes surface-enhanced Raman spectroscopy (SERS) coupled with a specifically designed aptamer, capable of binding to citrulline, conjugated to colloidal gold nanoparticles. The assay is then embedded within a vertical flow paper-fluidic platform as a deliverable at the POC, and a handheld Raman spectrometer (638-nm excitation) was used to interrogate the sample. Results showed good dynamic range and specificity with an average $73 \%$ decrease in SERS signal intensity with increasing concentrations of citrulline (0 to $50 \mu \mathrm{M})$ in phosphate-buffered saline compared to its controls: glycine, glutamine, histidine, and valine, which showed less than $10 \%$ average decrease in the presence of $200 \mu \mathrm{M}$ of each analyte. Further, the limit of detection (LOD) within a chip was determined to be $0.56 \mu \mathrm{M}$, whereas the LOD across chips was below $10 \mu \mathrm{M}$. $\odot$ The Authors. Published by SPIE under a Creative Commons Attribution 4.0 Unported License. Distribution or reproduction of this work in whole or in part requires full attribution of the original publication, including its DOI. [DOI: 10.1117/1.JBO.24.12.127002]
\end{abstract}

Keywords: surface-enhanced Raman spectroscopy; paper fluidics; aptamer; point-of-care technology.

Paper 190231R received Jul. 3, 2019; accepted for publication Nov. 15, 2019; published online Dec. 9, 2019.

\section{Introduction}

The ability to detect small molecules, such as biomarkers, relies heavily on the methodology used to provide specific, sensitive, and repeatable identification of these molecules. Citrulline is one such small molecule biomarker that is of interest to clinicians, as changes in its circulating physiological blood levels $(\sim 40 \mu \mathrm{M})$ can indicate the presence of a certain disease relating to gut function. Citrulline is an amino acid, and researchers have shown that levels below $\sim 10 \mu \mathrm{M}^{1}$ can be an indicator of villous atrophy disease, whereas levels around $\sim 20 \mu \mathrm{M}$ could be a sign of short bowel syndrome ${ }^{1}$ or short bowel mass integrity relating to HIV-associated villous atrophy. ${ }^{2}$ Therefore, it is of interest to be able to detect and monitor this biomarker quantifiably. The current method in monitoring citrulline is carried out by collecting a sample of the patient's blood and sending it to a central laboratory to be analyzed via different chromatography and mass spectroscopy techniques. ${ }^{3,4}$ This is not desirable for rapid diagnostic and onsite monitoring at point-of-care (POC). Therefore, the design of a POC device deliverable at a doctor's office could potentially allow for routine monitoring of a patient's intestinal function and provide timely diagnosis and treatment.

Our group has previously reported a designed aptamer (H8DAE) that was synthesized to specifically bind to citrulline. ${ }^{5,6}$ The selectivity of this aptamer to citrulline was tested

*Address all correspondence to Gerard Coté, E-mail: gcote@tamu.edu against a cocktail of 20 essential amino acids using microscale thermophoresis (MST). Results showed a strong binding affinity of $\sim 5 \mu \mathrm{M}$ to citrulline and a weak binding affinity to the other amino acids $(\sim 250 \mu \mathrm{M})$ outside the physiological relevant concentration $(<40 \mu \mathrm{M})$. Aptamers are synthetic single-stranded DNA/RNA molecules that are designed to be more thermally stable than antibodies and can bind to its target with high specificity and affinity. ${ }^{5}$ One property of aptamers, which many researchers have reported, is the conformational change that an aptamer can undergo on binding to its target. ${ }^{7}$ In this study, it was observed that the H8DAE aptamer can carry out this conformation change on binding to citrulline. To monitor this behavior and utilize it for the tracking of changes in citrulline concentrations, the aptamer was modified at the $5^{\prime}$ end with a reporter molecule $(\mathrm{RM})$, Cyanine 3 (Cy3: extinction $\sim 554 \mathrm{~nm})$, and at the $3^{\prime}$ end with a functional thiol group for covalent binding to gold nanoparticles (AuNPs). The interaction between the $\mathrm{RM}$ and the AuNP as the aptamer changes its conformation creates a change in distance between the RM and the nanoparticle surface. This change can be monitored using surface-enhanced Raman spectroscopy (SERS) or fluorescence. However, given the higher enhancement factor of SERS over fluorescence resonance and narrower peaks allows for the signal to be differentiated more easily from background noise (e.g., the autoflourescence one might find in more complex samples) as well as the eventual potential for multiplexing due to narrower Raman peaks, the assay was optimized for SERS.

Raman spectroscopy utilizes one wavelength of excitation light and its inelastic scattering properties to provide a chemical 
fingerprint of molecules of interest. Particularly, SERS has allowed for the amplification of these signals, which can therefore provide a tool for tracking small molecules. SERS is an analytical tool that utilizes plasmonic substrates (i.e., gold, silver, copper, etc.) and Raman active molecules to provide a chemical and electromagnetic enhancement on the order of $10^{6}$ to $10^{8}$ times the raw Raman signal of that molecule. ${ }^{8-11}$ The enhancement relies on the molecule of interest being in close proximity to substrate's surface $(<3 \mathrm{~nm})$ and the excitation source being in resonance with the local surface plasmons of the substrate. ${ }^{12}$ Recent advancements in the design of portable and handheld Raman spectrometers have allowed for the use of this modality in the field at the POC. Furthermore, this optical modality can be coupled with a paper-fluidic platform for easy fabrication and amplification of the Raman active molecule and aptamer to generate a low-cost chip for rapid detection of citrulline.

The application of SERS-based paper fluidics for disease diagnosis is a growing field with researchers utilizing the simplicity and cost effectiveness of paper fluidics to design disposable cartridges that can detect multiple analytes under Raman spectroscopy, with better quantification and sensitivity than the well-known colorimetric paper assays. ${ }^{13-16}$ Typically, these paper-fluidic membranes are cellulose-based and allow for the flow or immobilization of SERS-active nanoparticles within its fiber matrix. Recently, Hwang et al. ${ }^{13}$ demonstrated the use of lateral flow paper fluidics for the detection of HIV-1 DNA, thyroid-stimulating hormone, and an exotoxin. ${ }^{14}$ Other groups have utilized SERS-based lateral flow systems in the design of rapid diagnostic disposable chips. ${ }^{15,16}$ Although lateral flow designs are more commonly used in developing these SERS paper-based sensors and in commercial rapid diagnostic sticks, they are hindered by low sensitivity, large sample volume needed to drive the fluid the full length of the sensor, reaction time, and line interference, which makes it difficult to use multiple lines for multiplexing. ${ }^{17}$ In contrast, paper fluidics in the form of vertical flow can allow for relatively low sample volume and improved sensitivity, as a smaller distance is required for fluid to travel during the filtration and concentration stages, amounting in lower loss of analyte in the paper before arrival at the detection zone. ${ }^{18}$ Also, to some extent, the flow rate and, in turn, the reaction time of the assay can be better controlled in a vertical flow system by controlling the area through which the fluid will flow.

Herein, a vertical flow platform was used where the SERSactive nanoparticles were immobilized on nitrocellulose (NC) paper, and wicking membranes of varying pore sizes and hydrophilic area were used to direct the flow of the analyte solution through the SERS-active region toward the waste pad. The SERS-active nanoparticles were comprised of a Raman reporter-labeled aptamer (Cy3-H8DAE) conjugated on AuNPs. It is expected that the SERS signal intensity will change as the aptamer binds to the citrulline analyte and change its conformation. This change in conformation increases the distance between the Raman active molecule and the nanoparticle surface, thus impacting the chemical and electromagnetic enhancement effect resulting in a gradual change in SERS intensity with increasing concentration of the analyte.

The potential use of this SERS-based vertical flow assay was investigated to monitor its response as a function of biomarker concentration when measured using a portable handheld Raman spectrometer $(638 \mathrm{~nm} ; 40 \mathrm{~mW})$.

\section{Materials and Methods}

\subsection{Materials}

The aptamer (H8DAE) was synthesized, characterized using MST, and purchased from Base Pair Biotechnologies (Pearland, Texas). The NC membrane (pore size of $0.45 \mu \mathrm{m}$ ) and blotting absorbent filter paper (\# 1703960) were purchased from BioRad (Hercules, California), and the pure cellulose chromatography paper (Cat. No. 05714-1) was acquired from Fisher Scientific (Waltham, Massachusetts). For the synthesis of the citratecapped AuNPs, gold (III) chloride $\left(\mathrm{HAuCl}_{4}\right)$ and sodium citrate were obtained from Sigma-Aldrich (St. Louis, Missouri). Before conjugation of the thiolated aptamer, the disulfide bonds were reduced using tris(2-carboxyethyl)phosphine hydrochloride (TCEP) purchased from Sigma-Aldrich (St. Louis, Missouri). The immobilization of the AuNPs on the nitrocellulose membrane was performed using streptavidin, which was purchased from VWR (Radnor, Pennsylvania) and a PEGylated-biotin linker was obtained from ThermoFisher Scientific (Waltham, Massachusetts) and modified accordingly. All experiments were performed in $1 \times$ phosphate-buffered saline $(1 \times \mathrm{PBS})$ at $\mathrm{pH} 7.4$ and Raman measurements were taken using an ID Raman mini 2.0 (Metrohm-formally purchased from Ocean Optics, Switzerland).

\subsection{Aptamer-Conjugated Gold}

The 34-nm citric acid-capped AuNPs were prepared using a well-known seed-mediated method adopted from Bastús et.al. ${ }^{19}$ Briefly, a solution of gold seed was prepared by pipetting $335 \mu \mathrm{L}$ of $25 \mathrm{mM} \mathrm{HAuCl}_{4}$ solution into $50 \mathrm{ml}$ of sodium citrate solution $(2.2 \mathrm{mM})$ being heated at $100^{\circ} \mathrm{C}$. After $\sim 15 \mathrm{~min}$ of reaction, the solution was cooled to $90^{\circ} \mathrm{C}$ for kinetically controlled shell growth. To grow the nanoshell further to the desired size, $335 \mu \mathrm{L}$ of $60 \mathrm{mM}$ sodium citrate solution was mixed with the gold solution $\left(90^{\circ} \mathrm{C}\right)$ for 2 min followed by an additional pipetting of $25 \mathrm{mM} \mathrm{HAuCl}{ }_{4}$ solution $(335 \mu \mathrm{l})$ to the solution. Afterward, the reaction was allowed to occur for $30 \mathrm{~min}$. The size of the particles was confirmed via UV-Vis spectroscopy and the table provided by Haiss et al. ${ }^{20}$ Additional cycles of $335 \mu \mathrm{L}$ of $60 \mathrm{mM}$ sodium citrate solution followed by $25 \mathrm{mM}$ $\mathrm{HAuCl}_{4}$ solution $(335 \mu \mathrm{l})$ after 2 min were repeated until the desired size was obtained.

Following AuNP synthesis, the thiolated, fluorescently labeled aptamer was conjugated on its surface in a 9200:1 molar ratio. Briefly, the aptamers' disulfide bonds were reduced to generate functional thiol groups by incubating the aptamers $(100 \mu \mathrm{M})$ in equal volume of $20 \mathrm{mM}$ TCEP solution for an hour. Following this incubation, the TCEP was removed via $3 \mathrm{kDa}$ Nanosep centrifuge filters and resuspended in $1 \times$ PBS. The final concentration of the aptamers was determined via UV-Vis spectroscopy. The aptamers were then folded into their tertiary state by heating the solution to $93^{\circ} \mathrm{C}$ for $5 \mathrm{~min}$ and allowing this solution to slowly cool to room temperature. After folding, the aptamers were added to the gold colloidal solution at a 9200:1 ratio. This was allowed to shake for an hour and then incubated overnight at room temperature to ensure proper time was given for the thiolated aptamers to adhere to the nanoparticle surface. Following this reaction, $4.5 \mathrm{mM}$ citrate buffer ( $\mathrm{pH} 3$ ) was added to the solution and allowed to shake for $10 \mathrm{~min}$ before centrifugation. Thiol-pegylated biotin $(200 \mu \mathrm{M})$ was added at 1000:1 molar ratio and allowed to shake for $10 \mathrm{~min}$ before centrifuging 
(1.2 krcf) for $10 \mathrm{~min}$ to remove any unconjugated chemistry. The newly conjugated particles were resuspended to a final AuNP probe concentration of $0.5 \mathrm{nM}$ in distilled water for later characterization and use.

\subsubsection{Stability and functionality characterization of conjugated particles}

The successful conjugation and stability of the aptamer-conjugated particles were determined via zeta-potential measurements. Typically, colloidal nanoparticles with surface charge less than $-25 \mathrm{mV}$ or greater than $+25 \mathrm{mV}$ are said to be relatively stable. ${ }^{21}$ Therefore, to confirm the stability of the particles, $50 \mu \mathrm{l}$ of bare citrate-capped AuNPs and $50 \mu \mathrm{l}$ of aptamerconjugated AuNPs probes were added to separate $950 \mu \mathrm{l}$ distilled water and then loaded into a zeta cell (Malvern \# DTS1070) via a 1-ml disposable syringe. Measurements via a Zetasizer Nano ZS90 (Malvern, United Kingdom) were taken with 10 scans per measurement and 3 measurements per sample.

\subsection{Vertical Flow Paper Fluidics}

After determining the functionality of the sensor via fluorescence in free solution, a paper-fluidic platform was developed for POC testing of the citrulline biomarker. The well-established vertical flow paper (VFP) fluidics platform was adopted for this design. Specifically, the paper-fluidic device was designed as shown in Fig. 1 where three different paper membranes of varying pore sizes were used to (1) immobilize the probes, (2) wick the fluid as it flows vertically through to the final paper used to (3) absorb the waste solution (in a blotting absorbent pad). First, NC paper of 450-nm pore size was chosen for probe immobilization. The membrane was cut into a 5-mm-diameter circle using a biopsy punch. Streptavidin $\left(2 \mathrm{mg} / \mathrm{ml}\right.$ in $\left.\mathrm{DI} \mathrm{H}_{2} \mathrm{O}\right)$ was then incubated on its surface by pipetting $2 \mu \mathrm{l}$ of this solution to the membrane, immediately followed by $1 \mu \mathrm{l}$ of PBS and $5 \mu \mathrm{l}$ of the biotinylated AuNPs probes solution. Enough time was given for the membrane to dry $(\sim 1 \mathrm{~h})$. Next, a hydrophobic barrier was created on the wicking paper (i.e., pure cellulose membrane) by curing polydimethylsiloxane in the designated dark blue areas seen in Fig. 1. The three papers were then assembled together as depicted in Fig. 1 using 3M double-sided adhesive tape (\#444).

\subsection{SERS Response to Citrulline}

The SERS response of the assay to varying concentrations of citrulline was observed using a portable handheld Raman spectrometer at $638-\mathrm{nm}$ excitation and $40-\mathrm{mW}$ power. First, the SERS signal of the membrane in its dry state was measured as baseline. Then $10 \mu \mathrm{l}$ of PBS was added to the membrane at the sample loading zone and Raman measurements were taken after

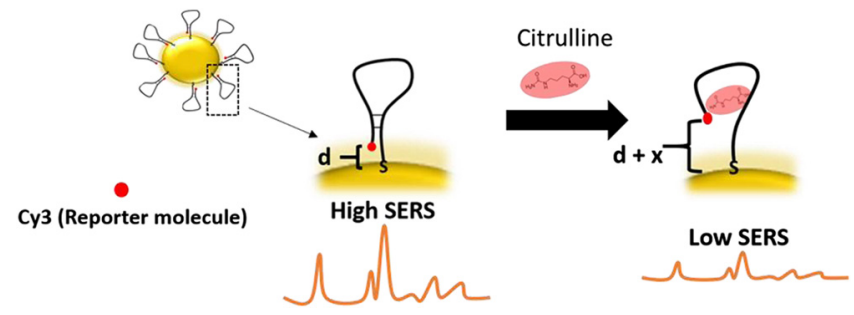

Fig. 1 Schematic of citrulline SERS bioassay.

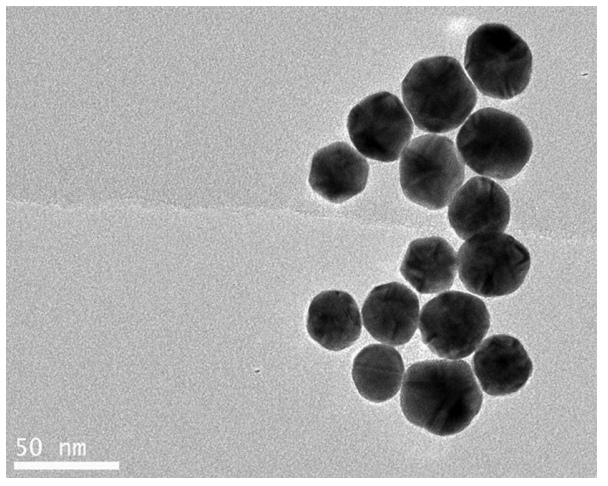

Fig. 2 TEM images of bare citrate-capped AuNPs ( 34 nm).

$20 \mathrm{~min}$. Then $10 \mu \mathrm{l}$ of $10 \mu \mathrm{M}$ of citrulline was added to the same zone and after equal amount of time another Raman spectrum was taken. This was repeated for $0,10,20,40$, and $50 \mu \mathrm{M}$ concentrations. This experiment was performed in triplicate.

To determine the feasibility of these chips to be used as one-time disposable chips, the different concentrations of citrulline were tested on separate chips. Again, the SERS signal of the membrane in its dry state was measured as baseline. Then $10 \mu \mathrm{l}$ of PBS (i.e., $0 \mu \mathrm{M}$ citrulline) was added to the membrane at the sample loading zone and Raman measurements were taken after $20 \mathrm{~min}$. Then $10 \mu \mathrm{l}$ of $10 \mu \mathrm{M}$ of citrulline was added to the same zone and after equal amount of time another Raman spectrum was taken. This was repeated for citrulline concentrations of 20, 40, and $50 \mu \mathrm{M}$ all on separate chips. This experiment was repeated in triplicates for citrulline concentrations of $0,10,20$, 40 , and $50 \mu \mathrm{M}$, where the $0 \mu \mathrm{M}$ was used as the baseline normalization factor for each chip.

\subsection{Selectivity of the SERS-Based Paper-Fluidic Assay}

The selectivity of the SERS-based paper-fluidic assay was investigated by comparing the percentage of the SERS intensity signal in the presence of $200 \mu \mathrm{M}$ citrulline and three noncomplementary amino acids: valine (Val), histidine (His), and glycine (Gly), all at $200 \mu \mathrm{M}$ concentration. Like before, the paper-fluidic chip was interrogated using the Ocean Optics portable handheld Raman spectrometer at $638-\mathrm{nm}$ excitation and 40-mW power. First, the SERS signal of each membrane in its dry state was measured as baseline. Then $10 \mu \mathrm{l}$ of PBS was added to the membrane at the sample loading zone and Raman measurements were taken after $20 \mathrm{~min}$. Then $10 \mu \mathrm{l}$ of the specific amino acid $(200 \mu \mathrm{M})$ was added to the same zone and after an equal amount of time another Raman spectrum was taken. This was repeated for two more times for each amino acid.

\section{Results and Discussion}

\subsection{Characterization of Aptamer-Conjugated Gold}

The Cy3-labeled aptamer was conjugated on the surface of AuNPs to cause a distance-dependent change in the signal as depicted in Fig. 1. The size of the gold was measured via absorbance and confirmed by TEM images to be $\sim 34 \mathrm{~nm}$ in diameter (Fig. 2). The conjugation of these particles was verified via absorbance measurements and resulted in a 2-nm redshift change between bare citrate-capped AuNPs and the aptamerconjugated AuNPs (Fig. S1 in the Supplementary Material). 


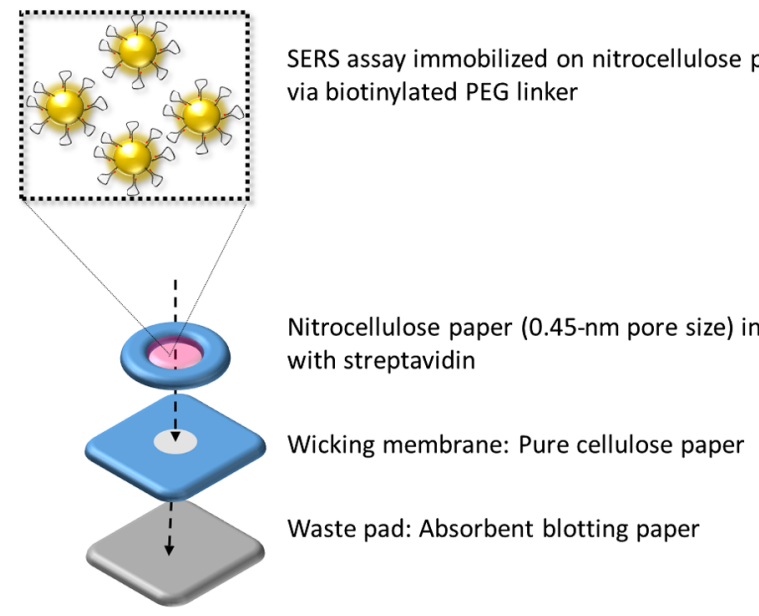

Fig. 3 Schematic of VFP chips immobilized with aptamer-conjugated SERS-active probes for citrulline detection.

This shift is a result in the change of the particles' dielectric constant due to the presence of the thiolated aptamer covalently bound to the particles' surface, thus resulting in a change in localized surface plasmon resonance and confirming successful conjugation. This conjugation was further confirmed by the change in zeta potential of the particle surface of citrate-capped AuNPs $(-42+0.9 \mathrm{mV})$ and aptamer-AuNPs $(-36+0.67 \mathrm{mV})$. Furthermore, the obtained surface charge of less than $-25 \mathrm{mV}$ indicates the stability of both particles. ${ }^{21}$

\subsection{SERS in Response to Citrulline Concentration}

To create a portable platform to collect and concentrate the citrulline analyte and to amplify the SERS signal, a VFP fluidic chip was created (Fig. 3).

Following the immobilization of the probes on the nitrocellulose membrane and the assembly of the VFP chip, the SERS signal was measured for varying concentrations of citrulline. First, the chips were tested by varying the concentration of citrulline from 0 to $50 \mu \mathrm{M}$ [Fig. 4(a)] within the same chip to determine the conformational change and saturation limit of the assay. Figure 4(a) shows the SERS response decreasing with increasing concentration of the analyte, which indicated the movement of the dye away from the nanoparticle surface (i.e., outside of the electromagnetic field) as citrulline binds

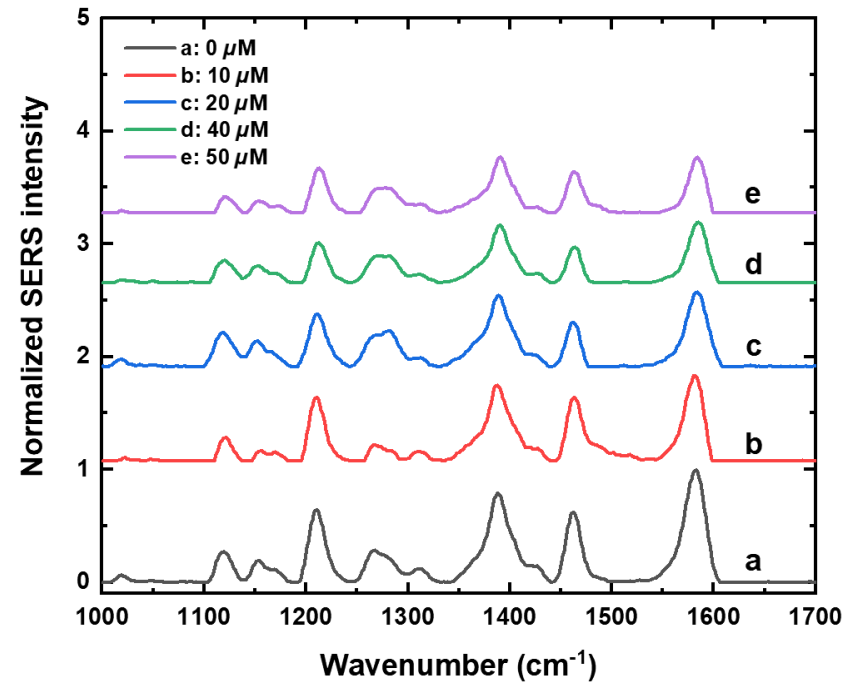

Fig. 5 SERS response on the VFP chip in the presence of different concentrations of citrulline in PBS across different chips (all data normalized to its $0 \mu \mathrm{M}$ concentration).

to the aptamer and the aptamer changes its conformation in response to this binding. The bands near 1197, 1390, 1468, and $1585 \mathrm{~cm}^{-1}$ are indicative of the bands for Cy 3 and not of the Raman bands of the DNA aptamer or citrulline. ${ }^{22,23}$ The limit of detection (LOD) for the VFP chip was determined to be $0.56 \mu \mathrm{M}$ (Fig. S2 in the Supplementary Material). This detection limit is well below the physiological relevant concentrations needed to determine disease state of the gut. Furthermore, the small error bars indicate the ability to be able to reproduce this response across different chips $(n=3)$ with a significant statistical difference among concentrations $\leq 40 \mu \mathrm{M}$ ( $p=$ $0.00015)$, thus indicating the potential use of this paper-based assay for the detection of low levels of citrulline relating to diseases such as villous atrophy and short bowel syndrome.

The chips were tested for the ability to be used as a disposable, one-time use lab on a chip platform. To accomplish this, different chips were loaded with different concentrations of the target with different citrulline concentrations $(0,10,20,40$, and $50 \mu \mathrm{M}$ ). Due to fluctuating baseline from chip to chip, all chips were first measured with $0 \mu \mathrm{M}$ solution (i.e., PBS) before the desired concentration was added and final measurements were taken at that concentration. The data for each chip were then
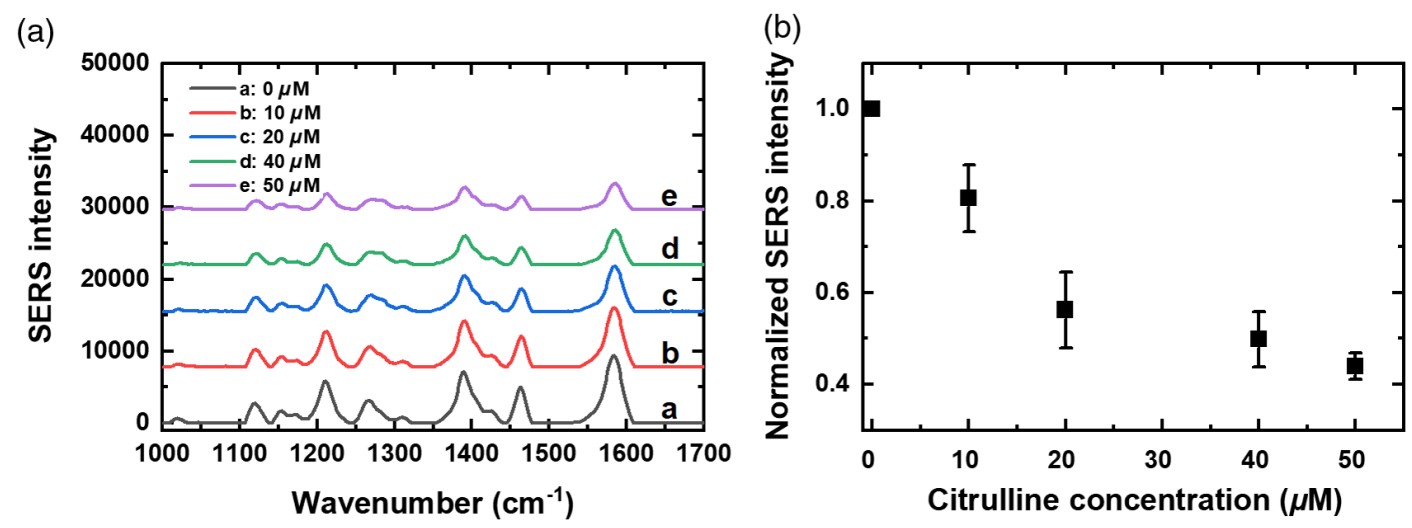

Fig. 4 (a) SERS response on the VFP chip in the presence of varying concentrations of citrulline within the same chip. (b) Calibration curve for the average of three different chips at $1389 \mathrm{~cm}^{-1}$ peak. 


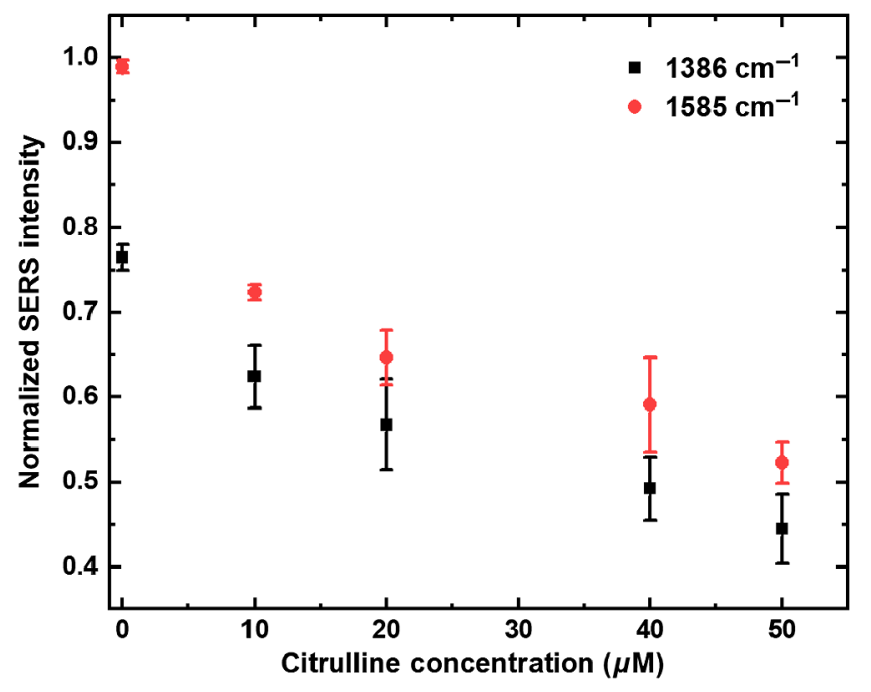

Fig. 6 Calibration curve at 1386 and $1585 \mathrm{~cm}^{-1}$ peak for the average of three sets of disposable chips incubated with different concentrations of citrulline.

normalized to its zero concentration to account for this variation. Similar to what was observed in Fig. 4, the SERS response indicated a decrease in signal intensity with higher concentrations of the citrulline target (Fig. 5).

The LOD was calculated as 9.5 and $7.8 \mu \mathrm{M}$ for 1386 and $1585 \mathrm{~cm}^{-1}$, respectively (Fig. S3 in the Supplementary Material). This LOD is again well below the physiological relevant concentration of $40 \mu \mathrm{M}$ indicating that the VFP assay's ability to detect low concentrations of citrulline necessary to make a diagnosis of disease of the gut. The upper limit of quantification, defined as the maximum detection that would result in a true positive, was calculated to be 79.6 and $58.8 \mu \mathrm{M}$ for 1386 and $1585 \mathrm{~cm}^{-1}$, respectively. A calibration of three different chips exposed to the same concentration of citrulline (Fig. 6) shows the reproducibility of the assay (denoted by the error bars). To assess the precision of the chips, the mean absolute difference was calculated to be $3.3 \%$ and $2.3 \%$ for 1386 and $1585 \mathrm{~cm}^{-1}$, respectively. According to literature, citrulline concentrations $<30 \mu \mathrm{M}$ indicate a certain level of disease state of the gut. This assay is able to detect changes in levels around that concentration; however, the performance can be improved by changing the detection platform. One limitation of paper fluidics is the inability to control the rate at which it dries, which in turn may cause baseline drift from chip to chip. One potential way to overcome this issue is immobilizing this assay in a microfluidic device or using a combination vertical and lateral flow paper with an optically transparent cover over the probe area of the paper, which can create a stable fluid environment for the assay and allow for optimum observation of the conformational change that the aptamer undergoes in the presence of citrulline.

\subsection{Selectivity of the SERS in Response to Citrulline}

To determine the aptamer selectivity to citrulline, three other nonspecific amino acids, histidine (His), valine (Val), and glycine (Gly), were tested on the paper-fluidic device at high concentrations $(200 \mu \mathrm{M})$. This concentration was selected based on previously reported average blood plasma concentrations of these amino acids: His $(83 \mu \mathrm{M})$, Val $(237 \mu \mathrm{M})$, and Gly

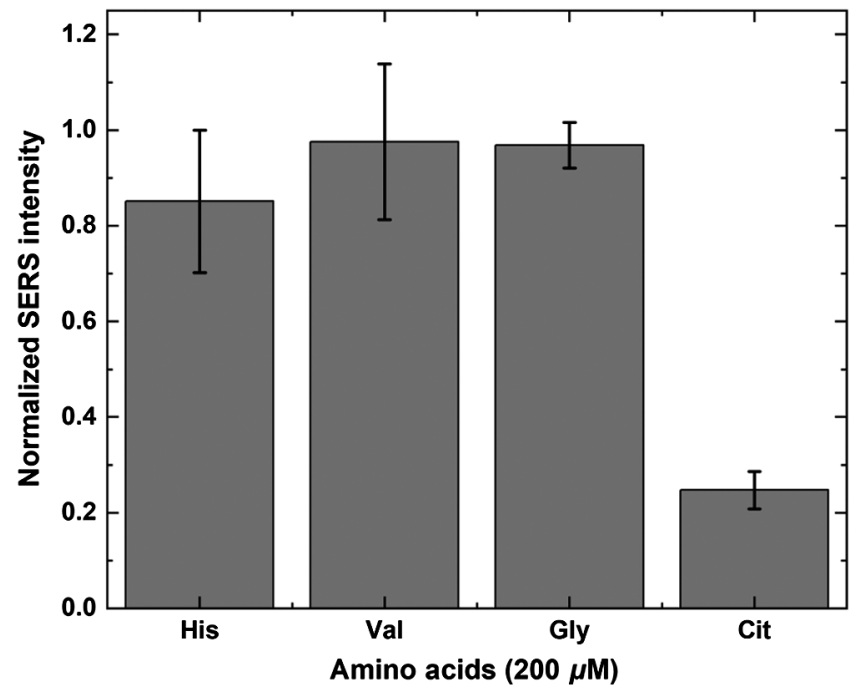

Fig. 7 Normalized SERS intensity at $1585 \mathrm{~cm}^{-1}$ of histidine (His), valine (Val), and glycine (Gly) compared to citrulline (Cit) at high concentrations demonstrating VFP assay selectivity.

$(224 \mu \mathrm{M}) .^{4}$ Figure 7 shows the change in the normalized SERS intensity at $1585 \mathrm{~cm}^{-1}$ of the assay in the presence of $200 \mu \mathrm{M}\left(I_{200}\right)$. The data were normalized to the assay initial exposure to only PBS $\left(I_{0}\right)$ prior to the addition of the amino acid. The percentage decrease for each amino acid was calculated as $\left(I_{200} / I_{0} \times 100\right)$ and results indicated a percentage of change of $15 \%, 2 \%, 3 \%$, and $75 \%$ for His, Val, Gly, and Cit, respectively. Although His showed a $15 \%$ change for the assay exposed to $200 \mu \mathrm{M}$, it is typically found in blood plasma around $83 \mu \mathrm{M}$; therefore, this percentage change is not likely to be evident and thus affecting the sensing of citrulline.

\section{Conclusion}

This work takes advantage of the conformational change of aptamer H8DAE on binding to citrulline to detect different concentrations of this analyte around its physiological relevant value. Concentrations below $30 \mu \mathrm{M}$ have been reported to be indicative of intestinal disease and the SERS-based paper-fluidic chip, proposed herein, was able to detect low concentrations of the analyte with an LOD below $10 \mu \mathrm{M}$. However, the sensitivity between 20 and $40 \mu \mathrm{M}$ was low and may be better optimized with a combination vertical and lateral flow paper or microfluidic device. Overall, this work showed that an aptamer-SERS assay can potentially be used as a platform to collect and analyze the amino acid, citrulline.

\section{Disclosures}

The authors have no relevant financial interests in this article and no potential conflicts of interest to disclose.

\section{References}

1. P. Crenn, B. Messing, and L. Cynober, "Citrulline as a biomarker of intestinal failure due to enterocyte mass reduction," Clin. Nutr. 27(3), 328-339 (2008).

2. C. Papadia et al., "Plasma citrulline as a quantitative biomarker of HIVassociated villous atrophy in a tropical enteropathy population," Clin. Nutr. 29(6), 795-800 (2010). 
3. P. N. Demacker et al., "Plasma citrulline measurement using UPLC tandem mass-spectrometry to determine small intestinal enterocyte pathology," J. Chromatogr. B 877(4), 387-392 (2009).

4. T. Terrlink, P. Van Leeuwen, and A. Houdijk, "Plasma amino acids determined by liquid chromatography within 17 minutes," Clin. Chem. 40(2), 245-249 (1994).

5. B. M. Walton et al., "Surface-enhanced Raman spectroscopy competitive binding biosensor development utilizing surface modification of silver nanocubes and a citrulline aptamer," J. Biomed. Opt. 22(7), 075002 (2017).

6. A. Locke, N. Deutz, and G. Coté, "Development of a paper-based vertical flow SERS assay for citrulline detection using aptamer-conjugated gold nanoparticles," Proc. SPIE 10501, 1050105 (2018).

7. D. Ogasawara et al., "Detection system based on the conformational change in an aptamer and its application to simple bound/free separation," Biosens. Bioelectron. 24(5), 1372-1376 (2009).

8. K. Kneipp et al., "Single molecule detection using surface-enhanced Raman scattering (SERS)," Phys. Rev. Lett. 78(9), 1667-1670 (1997).

9. T. Vo-Dinh et al., "Plasmonic nanoprobes: from chemical sensing to medical diagnostics and therapy," Nanoscale 5(21), 10127-10140 (2013).

10. E. C. Le Ru and P. G. Etchegoin, "Single-molecule surface-enhanced Raman spectroscopy," Аnnu. Rev. Phys. Chem. 63, 65-87 (2012).

11. W. E. Doering and S. Nie, "Single-molecule and single-nanoparticle SERS: examining the roles of surface active sites and chemical enhancement," J. Phys. Chem. B 106(2), 311-317 (2002).

12. P. L. Stiles et al., "Surface-enhanced Raman spectroscopy," Annu. Rev. Anal. Chem. 1, 601-626 (2008).

13. J. Hwang, S. Lee, and J. Choo, "Application of a SERS-based lateral flow immunoassay strip for the rapid and sensitive detection of staphylococcal enterotoxin B," Nanoscale 8(22), 11418-11425 (2016).

14. X. Fu et al., "A SERS-based lateral flow assay biosensor for highly sensitive detection of HIV-1 DNA," Biosens. Bioelectron. 78, 530-537 (2016).

15. E. P. Hoppmann, W. Y. Wei, and I. M. White, "Highly sensitive and flexible inkjet printed SERS sensors on paper," Methods 63(3), 219-224 (2013).

16. H. Torul et al., "Paper membrane-based SERS platform for the determination of glucose in blood samples," Anal. Bioanal. Chem. 407(27), 8243-8251 (2015).

17. Y. K. Oh et al., "Vertical flow immunoassay (VFA) biosensor for a rapid one-step immunoassay," Lab Chip 13(5), 768-772 (2013).

18. O. Clarke et al., "Development of a SERS-based rapid vertical flow assay for point-of-care diagnostics," Anal. Chem. 89(3), 1405-1410 (2017).

19. N. G. Bastús, J. Comenge, and V. Puntes, "Kinetically controlled seeded growth synthesis of citrate-stabilized gold nanoparticles of up to
$200 \mathrm{~nm}$ : size focusing versus Ostwald ripening," Langmuir 27(17), 11098-11105 (2011).

20. W. Haiss et al., "Determination of size and concentration of gold nanoparticles from UV-vis spectra," Anal. Chem. 79(11), 4215-4221 (2007).

21. nanoComposix, "Zeta potential analysis of nanoparticles," 1.1 (2012).

22. J. Qi et al., "Label-free, in-situ SERS monitoring of individual DNA hybridization in microfluidics," Nanoscale 6, 8521-8526 (2014).

23. I. Notingher, "Raman spectroscopy cell-based biosensors," Sensors 7(8), 1343-1358 (2007).

Andrea Locke is currently an Academic Pathways Fellow at Vanderbilt University. She worked as a research assistant engineer with the Center for Remote Health Technologies and Systems at Texas A\&M University. She received her PhD in biomedical engineering from Texas A\&M University in 2016. Her research interest is in developing point-of-care (POC) technologies, particularly for use in low-resource settings. Currently, her focus is on investigating biophotonics for the detection of biomarkers indicative of infection and inflammation.

Sayali Belsare is a third-year PhD student in the Department of Biomedical Engineering at Texas A\&M University. She works for Dr. Coté as a part of the Optical Bio-Sensing Lab. Her research involves the development of a paper-fluidic colorimetric system for monitoring gestational diabetes at the POC. She has been a member of the SPIE Texas A\&M student chapter since 2017 and has been in charge of their outreach activities since 2018.

Nicolaas Deutz currently serves as the director for the Center for Translational Research in Aging and Longevity at Texas A\&M University. For more than 30 years, his research background and expertise have focused on nutrition, metabolism, and physiology studies involving the use of stable isotope methodologies, both in humans and animals. He has extensive experience with using these methods in several studies to unravel the metabolic changes in patients with chronic diseases.

Gerard Coté is a Texas A\&M University Regents Professor and the James J. Cain Professor I in biomedical engineering at Texas A\&M University. His research includes biomedical sensing for diagnostic and monitoring applications with a recent focus on the development of wearable and handheld medical devices for underserved populations. He is a fellow of four societies (IEEE, SPIE, BMES, and AIMBE) and is an entrepreneur who has founded several start-up companies. 